\title{
A common ground for resource and welfare egalitarianism*
}

\author{
Juan D. Moreno-Ternero ${ }^{\dagger} \quad$ John E. Roemer ${ }^{\ddagger}$
}

October 27, 2010

\begin{abstract}
Resource egalitarianism and welfare egalitarianism are two focal conceptions of distributive justice. We show in this paper that they share a solid common ground. To do so, we analyze a simple model of resource allocation in which agents' abilities (to transform the resource into an interpersonally comparable outcome) and starting points may differ. Both conceptions of egalitarianism are naturally modeled in this context as two allocation rules. The two rules are jointly characterized by the combination of three appealing axioms: priority, solidarity, and composition.
\end{abstract}

Keywords: resource allocation, egalitarianism, welfare, priority, solidarity, composition. JEL Classification: D63

${ }^{*}$ We thank Marc Fleurbaey, François Maniquet, Hervé Moulin, an Advisory Editor of this journal, and two anonymous referees for helpful comments and suggestions. We also thank the audiences at the Third Workshop on Social Decisions (Málaga), the Ninth International Meeting of the Society for Social Choice and Welfare (Montreal), and the Workshop on Fairness, Equality of Opportunity and Public Economics (Louvain-laNeuve). Moreno-Ternero acknowledges financial support from the Spanish Ministry of Science and Innovation (ECO2008-03883) and from Junta de Andalucía (P08-SEJ-04154).

†Universidad de Málaga, Universidad Pablo de Olavide, and CORE, Université catholique de Louvain.

${ }_{\ddagger}^{\ddagger}$ Elizabeth S. and A. Varick Stout Professor of Political Science and Economics, Yale University. 


\section{Introduction}

A significant amount of effort in political philosophy over the past few decades has been concentrated on the issue of distributive justice. A central impetus for this should be attributed to John Rawls's theory (e.g., Rawls, 1971), which constituted an influential endorsement for egalitarianism. Egalitarian doctrines tend to express the idea that all human persons are equal in fundamental worth or moral status. In spite of this seemingly unquestionable idea, egalitarianism is a contested concept in social and political thought. Discussions in moral philosophy have offered us a wide menu in answer to the question: equality of what? (e.g., Sen, 1980). In other words, if one is an egalitarian, what should one wish to equalize? Two well-known (and polar) theories have been singled out to answer this question in distributive justice. Resource egalitarianism holds that a distributional scheme treats people as equals when it distributes or transfers so that no further transfer would leave their shares of the total resources more equal (e.g., Dworkin, 1981b). Welfare egalitarianism holds that it treats them as equals when it distributes or transfers so that no further transfer would leave them more equal in welfare (e.g., Dworkin, 1981a). It is plain that both theories will offer different advice in many concrete cases. Nevertheless, we show in this paper that they have a solid common ground, as we shall derive them as the only allocation methods satisfying several appealing principles.

More precisely, imagine the following basic problem. There is an amount of wealth to be allocated among individuals, each of whom possesses a capability to transform wealth into some given valued (but non-transferable) outcome, and the achievements of individuals, with regard to that outcome, are interpersonally comparable. Think, for instance, of life expectancy as a function of investment in health care. In resource allocation problems of this sort, if individuals have equal rights over resources, resource egalitarianism (i.e., to distribute the available resource equally among all agents) and welfare (or outcome) egalitarianism (i.e., to distribute the resource among the population so as to equalize, as much as possible, the outcomes among them) are usually two focal points of distribution. We show that in this context both rules can actually be characterized together by combining three axioms. More precisely, we will consider the so-called priority axiom (e.g., Moreno-Ternero and Roemer, 2006), which imposes a positive discrimination (but only to a certain extent) towards the less capable of transforming resource into outcome; the so-called solidarity axiom (e.g., Thomson, 1983; Roemer, 1986) formalizing the idea that changes on the number of individuals and the available wealth should affect all incumbent agents in the same direction; and the so-called composition axiom (e.g., Young, 
1988; Moulin, 2000) pertaining to the behavior of a rule with respect to tentative allocations based on an incorrect estimation of the available wealth. Our main result will say that the two rules described above are the only ones satisfying these three axioms.

The rest of the paper is organized as follows. Section 2 presents the model of resource allocation upon which we will base our analysis. Section 3 is devoted to the results. Section 4 summarizes the connections of our work with some related literature. Section 5 concludes. For a smooth passage, we defer some proofs and provide them in the appendix.

\section{The model}

Let II represent a population of agents (a set with an infinite number of members) who transform a resource, sometimes called wealth, into an objectively measurable (and interpersonally comparable) outcome, sometimes called welfare. For each $i \in \mathbb{I}$, let $u_{i}: \mathbb{R}_{+} \rightarrow \mathbb{R}_{+}$be the individual function that models this process. ${ }^{1}$ We assume that, for each $i, u_{i}$ is continuous, strictly increasing and unbounded. We also assume that $\mathcal{U}=\left\{u_{i}: i \in \mathbb{I}\right\}$ constitutes a sufficiently rich domain. More precisely, we assume that $\mathcal{U}$ contains all positive affine transformations and, for reasons that will become clear later in the text, that it is closed under horizontal translations. Formally, if $a \in \mathbb{R}_{++}, b \in \mathbb{R}_{+}$and $u: \mathbb{R}_{+} \rightarrow \mathbb{R}_{+}$is defined by $u(x)=a x+b$, for all $x \in \mathbb{R}_{+}$, then $u \in \mathcal{U} .^{2}$ Similarly, if $u \in \mathcal{U}, c \in \mathbb{R}_{+}$and $v: \mathbb{R}_{+} \rightarrow \mathbb{R}_{+}$is defined by $v(x)=u(x+c)$, for all $x \in \mathbb{R}_{+}$, then $v \in \mathcal{U}$. Note that $u_{i}(0) \geq 0$ denotes the outcome that agent $i$ can generate with zero wealth. These levels, to be interpreted as agents' starting points, may well differ. In the example of life expectancy as a function of investment in health care, mentioned above, this would mean that even though some agents might not survive without investment in their health care, some others might do so.

Let $\mathcal{I}$ be the family of all finite subsets of $\mathbb{I}$. We define an economy $e$ as a triple $(N, u, W)$, where $N=\left\{i_{1}, i_{2}, \ldots, i_{n}\right\} \in \mathcal{I}$ is the set of agents, $u=\left(u_{i}\right)_{i \in N}$ is the profile of their outcome functions (defined as above), and $W \in \mathbb{R}_{+}$represents the available wealth. The family of all

\footnotetext{
${ }^{1}$ The main mathematical conventions and notations, used here, are as follows. The set of non-negative (positive) real numbers is $\mathbb{R}_{+}\left(\mathbb{R}_{++}\right)$. Vector inequalities are denoted by $>$and $\geq$. More precisely, $x>y$ means that each coordinate of $x$ is greater than the corresponding coordinate of $y$, whereas $x \geq y$ allows some of them to be equal. Finally, given a set $N$ and a subset $M$, we denote the projection of the vector $v \in \mathbb{R}_{+}^{|N|}$ over $M$ as $v_{M}$, i.e., $v_{M}=\left(v_{i}\right)_{i \in M}$.

${ }^{2}$ In particular, this implies that the graphs of all admissible outcome functions will cover the positive orthant, as well as the non-negative vertical axis.
} 
economies is $\mathcal{E}$.

An allocation rule, or simply a rule, is a function $R$ that associates to each economy an allocation indicating how to distribute the wealth available in the economy among its members. Formally, $R: \mathcal{E} \rightarrow \mathbb{R}_{+}^{n}$, where, for each $e=(N, u, W) \in \mathcal{E}, R(e)=\left(R_{i}(e)\right)_{i \in N} \in \mathbb{R}_{+}^{n}$ is such that $\sum_{i \in N} R_{i}(e)=W$. As we discard all information that is not contained in the description of an economy, we implicitly assume that rules are anonymous. In other words, the identity of agents will not play a role in the allocation process and we shall only focus on the outcome functions and the available wealth of the economy.

Examples of rules are the following. First, the rule that awards each agent the same amount: Resource-Egalitarian rule ( $R E): R E_{i}(N, u, W)=\frac{W}{n}$.

An alternative to the resource-egalitarian rule is obtained by focusing on the levels of outcome agents achieve, as opposed to the resources they receive, and choosing the vector at which these outcome levels are as equal as possible. In other words, the wealth is allocated initially to the agent(s) with the lowest starting point until her (their) outcome(s) become equal to the starting point(s) of the agent(s) with the second lowest starting point. Then, the rest of the wealth is distributed between these agents in a way to equalize their outcomes, until their outcomes are equal to the starting point(s) of the agent(s) with the third lowest starting point and so on. Formally,

Constrained Outcome-Egalitarian rule (COE): $C O E_{i}(N, u, W)=u_{i}^{-1}(\lambda)$ if $\lambda \geq u_{i}(0)$, and 0 otherwise, where $\lambda>0$ is chosen so that $\sum_{i \in N} C O E_{i}(N, u, W)=W$.

In other words, for each economy $(N, u, W)$, the constrained outcome-egalitarian rule determines a set $N_{1} \subseteq N$ such that, for all $i, j \in N_{1}$ and $k \in N \backslash N_{1}$,

$$
u_{i}\left(C O E_{i}(N, u, W)\right)=u_{j}\left(C O E_{j}(N, u, W)\right) \leq u_{k}(0)=u_{k}\left(C O E_{k}(N, u, W)\right)
$$

These two rules, which naturally translate the focal egalitarian theories we alluded to in the introduction, will be salient in our analysis. Nevertheless, many other rules can be defined. Instances would be dictatorial rules awarding a single agent the whole amount of wealth, proportional-like rules awarding wealth proportionally to some individual characteristic, or compromises between the above two arising from equalizing some combination between resources and outcome levels, as described next.

Formally, let $\Phi$ be the family of all functions $\varphi:\left\{\mathbb{R}_{+} \times \mathbb{R}_{++}\right\} \cup\{(0,0)\} \rightarrow \mathbb{R}_{+}$satisfying the following continuity and monotonicity assumptions: 


\section{Continuity:}

(i) $\varphi$ is continuous on $\mathbb{R}_{++}^{2}$, i.e.,

$$
\lim _{(x, y) \rightarrow\left(x_{0}, y_{0}\right)} \varphi(x, y)=\varphi\left(x_{0}, y_{0}\right), \text { for all }\left(x_{0}, y_{0}\right) \in \mathbb{R}_{++}^{2} .
$$

(ii) For all $y_{0} \in \mathbb{R}_{+}, \varphi(x, y)$ approaches $\varphi\left(0, y_{0}\right)$, as $(x, y)$ approaches $\left(0, y_{0}\right)$ along any curve $y=y(x)$ in $\mathbb{R}_{++}^{2}$, i.e.,

If $y_{0} \in \mathbb{R}_{+}$and $y: \mathbb{R}_{++} \rightarrow \mathbb{R}_{++}$is such that $\lim _{x \rightarrow 0^{+}} y(x)=y_{0}$ then $\lim _{x \rightarrow 0^{+}} \varphi(x, y(x))=\varphi\left(0, y_{0}\right)$.

\section{Monotonicity:}

(i) If $x \in \mathbb{R}_{+}$and $x^{\prime}, y \in \mathbb{R}_{++}$are such that $x<x^{\prime}$ then $\varphi(x, y) \leq \varphi\left(x^{\prime}, y\right)$.

(ii) If $x \in \mathbb{R}_{+}$and $y, y^{\prime} \in \mathbb{R}_{++}$are such that $y<y^{\prime}$ then $\varphi(x, y) \leq \varphi\left(x, y^{\prime}\right)$.

(iii) If $x \in \mathbb{R}_{+}$and $x^{\prime}, y, y^{\prime} \in \mathbb{R}_{++}$are such that $(x, y)<\left(x^{\prime}, y^{\prime}\right)$, then $\varphi(x, y)<\varphi\left(x^{\prime}, y^{\prime}\right)$.

(iv) $\varphi(0,0)=0 \leq \varphi(x, y)$ for all $(x, y) \in \mathbb{R}_{+} \times \mathbb{R}_{++}$.

Let $\varphi$ be a function in the class $\Phi$. For all $i \in \mathbb{I}$ define the function $\psi_{i}: \mathbb{R}_{+} \rightarrow \mathbb{R}_{+}$by $\psi_{i}(w)=\varphi\left(w, u_{i}(w)\right)$ for all $w \in \mathbb{R}_{+}$. It is straightforward to show that, for all $i \in \mathbb{I}, \psi_{i}$ is continuous and strictly increasing. Then, we define the family of constrained index-egalitarian rules as follows:

Constrained Index-Egalitarian rules $\left(\left\{E^{\varphi}\right\}_{\varphi \in \Phi}\right): E_{i}^{\varphi}(N, u, W)=\left(\psi_{i}\right)^{-1}(\xi)$ if $\xi \geq \psi_{i}(0)$, and 0 otherwise, where $\xi>0$ is chosen so that $\sum_{i \in N} E_{i}^{\varphi}(N, u, W)=W$.

In words, for each $\varphi \in \Phi$, and $e \in \mathcal{E}, E^{\varphi}(e)$ is the wealth allocation that lexicographically maximizes the $\varphi$-value across agents in $e$. More precisely, if agents are ranked according to the $\varphi$-value from their starting points, then the wealth is allocated initially to the agent with the lowest one until the resulting $\varphi$-value becomes equal to the second lowest (starting point) $\varphi$ value. Then, the rest of the wealth is distributed between these two agents in a way to equalize their $\varphi$-values, until these are equal to the third lowest (starting point) $\varphi$-value and so on. Equivalently, for each economy $(N, u, W)$, the constrained index-egalitarian rule determines a set $N_{1} \subseteq N$ such that, for all $i, j \in N_{1}$ and $k \in N \backslash N_{1}$,

$$
\psi_{i}\left(E_{i}^{\varphi}(N, u, W)\right)=\psi_{j}\left(E_{j}^{\varphi}(N, u, W)\right) \leq \psi_{k}(0)=\psi_{k}\left(E_{k}^{\varphi}(N, u, W)\right) .
$$

Note that, applied in this manner to an agent's wealth and outcome, $\varphi$ can be considered as a generalized index of wealth and outcome. So the rules just defined leximin a generalized index of wealth and outcome. It is straightforward to show that each rule $E^{\varphi}$ actually equalizes 
the corresponding index $\varphi$, when restricted to economies in which agents generate zero welfare with zero wealth. Hence, this family is a generalization to this context of the family introduced in Moreno-Ternero and Roemer (2006). ${ }^{3}$ It is also straightforward to show that the resourceegalitarian rule and the constrained outcome-egalitarian rule are members of the family of constrained index-egalitarian rules. Formally, if $\varphi(x, y)=x$, for all $(x, y) \in \mathbb{R}_{+}^{2}$, then $E^{\varphi} \equiv R E$, whereas if $\varphi(x, y)=y$, for all $(x, y) \in \mathbb{R}_{+}^{2}$, then $E^{\varphi} \equiv C O E$.

We now present several axioms for allocation rules that we endorse. We begin by introducing our axiom of priority, which says that no agent can dominate another agent both in resources and outcome.

Priority (PR). Let $e=(N, u, W) \in \mathcal{E}$ and $i, j \in N$ be such that $R_{i}(e)<R_{j}(e)$. Then $u_{i}\left(R_{i}(e)\right) \geq u_{j}\left(R_{j}(e)\right)$.

Note that this axiom (first introduced under this form in Moreno-Ternero and Roemer, 2006) guarantees that agents with poor outcome functions receive at least as much wealth as agents with better outcome functions. In other words, priority implies the weak equity axiom, introduced by Sen (1973). On the other hand, priority also says that an agent with a poor outcome function is never allocated so much that her outcome level exceeds that of an agent with a better outcome function. ${ }^{4}$

Our next axiom, solidarity, says that the arrival of new agents, whether or not this is accompanied by a change in the available wealth, should affect all the incumbent agents in the same direction. Formally,

Solidarity $(S L)$. Let $e=(N, u, W) \in \mathcal{E}$ and $e^{\prime}=\left(N^{\prime}, u^{\prime}, W^{\prime}\right) \in \mathcal{E}$, such that $N \subseteq N^{\prime}$. Let $N_{1}=\left\{i \in N: R_{i}(e)>0\right\}$. Then, one of the following three statements holds:

$$
\begin{gathered}
\qquad R_{i}\left(e^{\prime}\right)=R_{i}(e), \text { for all } i \in N, \\
R_{i}\left(e^{\prime}\right) \geq R_{i}(e), \text { for all } i \in N \text {, and } R_{i}\left(e^{\prime}\right)>R_{i}(e) \text {, for all } i \in N_{1}, \\
R_{i}\left(e^{\prime}\right) \leq R_{i}(e), \text { for all } i \in N \text {, and } R_{i}\left(e^{\prime}\right)<R_{i}(e) \text {, for all } i \in N_{1} .
\end{gathered}
$$

\footnotetext{
${ }^{3}$ The model in Moreno-Ternero and Roemer (2006) did not allow for differences in initial starting points and, therefore, the analysis therein was restricted to the domain of economies in which agents generate zero welfare with zero wealth.

${ }^{4} \mathrm{~A}$ counterpart to this axiom in the theory of fairness is the so-called "no-domination" axiom (e.g., Thomson, 1983; forthcoming), which says that no agent receives more of all goods than some other agent.
} 
Our solidarity axiom is modeling the fact that agents cannot benefit from a change (either in the available wealth or in the number of agents) if someone else suffers from it. Related formulations of the solidarity notion abound in the literature (see Thomson (forthcoming) for a survey, and Maniquet and Sprumont (2010) for a recent instance). ${ }^{5}$ This axiom is equivalent to the combination of two axioms that appear frequently in the literature: resource monotonicity and consistency. Resource monotonicity (e.g., Roemer, 1986) says that when a bad or good shock comes to an economy, all its members should share in the calamity or windfall.

Resource monotonicity (RM). Let $e=(N, u, W)$ and $e^{\prime}=\left(N, u, W^{\prime}\right) \in \mathcal{E}$ be such that $W^{\prime}<W$. Let $N_{1}=\left\{i \in N: R_{i}(e)>0\right\}$. Then, $R_{i}\left(e^{\prime}\right) \leq R_{i}(e)$ for all $i \in N$, and $R_{i}\left(e^{\prime}\right)<R_{i}(e)$ for all $i \in N_{1}$.

Consistency has played a fundamental role in axiomatic analysis (see, e.g., Thomson (2007) and the literature cited therein) even though it is mostly an operational (rather than ethical) axiom. It says that if a sub-group of agents secedes with the resource allocated to it under $R$ then, in the smaller economy, $R$ allocates the resource in the same way. In that sense, consistency can be interpreted as a notion of stability.

Consistency $(C Y)$. Let $e=(N, u, W)$ and $e^{\prime}=\left(N^{\prime}, u^{\prime}, W^{\prime}\right) \in \mathcal{E}$ be such that $N^{\prime} \subset N$ and $W^{\prime}=\sum_{i \in N^{\prime}} R_{i}(e)$. Then $R_{i}(e)=R_{i}\left(e^{\prime}\right)$, for all $i \in N^{\prime}$.

Our final property pertains to the behavior of a rule with respect to tentative allocations based on an incorrect estimation of the available wealth. To motivate this property, imagine the following scenario: after having committed to divide the available wealth, one finds that the actual amount to divide is larger than was initially assumed. Then, two options are open: either the tentative division is cancelled altogether and the allocation for the actual economy is obtained directly, or we add to the initial commitment the result of applying the rule to the subsequent economy with the remaining amount and the adjusted individual outcome functions that would emerge after the initial allocation. ${ }^{6}$ The requirement of composition is that both ways of proceeding should result in the same allocations. Formally,

Composition $(C P)$. Let $e=(N, u, W) \in \mathcal{E}$. Let $W^{1}, W^{2} \in \mathbb{R}_{++}$be such that $W=W^{1}+W^{2}$ and $e^{1}=\left(N, u, W^{1}\right) \in \mathcal{E}$. For each $i \in N$, let $\widehat{u}_{i} \in \mathcal{U}$ be such that $\widehat{u}_{i}(x)=u_{i}\left(x+R_{i}\left(e^{1}\right)\right)$ for

\footnotetext{
${ }^{5}$ Solidarity properties with respect to population changes were indeed introduced by Thomson (1983). As mentioned above, our axiom is stronger as it refers to simultaneous changes in two parameters (namely, resources and population). Chun (1999) formulates a counterpart notion in the context of bankruptcy problems.

${ }^{6}$ This is the reason why we require $\mathcal{U}$ to be closed under horizontal translations.
} 
all $x \in \mathbb{R}_{+}$, and let $e^{2}=\left(\widehat{N},\left(\widehat{u}_{i}\right)_{i \in N}, W^{2}\right) \in \mathcal{E}$. Then, $R(e)=R\left(e^{1}\right)+R\left(e^{2}\right)$.

The property of composition has a relative in the theory of axiomatic bargaining: the so-called "step-by-step negotiations" axiom introduced by Kalai (1977), which considers two nested bargaining sets and uses the bargaining solution for the smaller bargaining set as the disagreement point for the larger bargaining set. It is the basis for the characterization of the egalitarian solution in such context. The same principle has also been frequently used in other related contexts (e.g., Young, 1988; Moulin, 2000; Moulin and Stong, 2002).

\section{The results}

As anticipated in the introduction, the main result of this paper shows that the resourceegalitarian rule and the constrained outcome-egalitarian rule are characterized by the axioms described above. In order to prove such result, we present first another result, which generalizes Theorem 1 in Moreno-Ternero and Roemer (2006).

Theorem 1 A rule defined on $\mathcal{E}$ satisfies priority and solidarity if and only if it is a constrained index-egalitarian rule.

The proof of Theorem 1 appears in the Appendix. Note that this theorem is indeed a generalization of Theorem 1 in Moreno-Ternero and Roemer (2006), which says that, for the domain of economies in which all agents generate zero welfare from zero wealth, the family of index-egalitarian rules is characterized by the corresponding axiom of restricted domain, the axiom of priority and the solidarity axiom.

We are then ready to state our main result.

Theorem 2 A rule defined on $\mathcal{E}$ satisfies priority, solidarity and composition if and only if it is either the resource-egalitarian rule or the constrained outcome-egalitarian rule.

Proof. By Theorem 1, we know that $R E$ and $C O E$ satisfy $P R$ and $S L$. It is straightforward to show that $R E$ satisfies $C P$. We then show that $C O E$ satisfies $C P$.

Let $e=(N, u, W) \in \mathcal{E}$. For ease of exposition, assume that $N=\{1,2, \ldots, n\}$ and that agents are ranked (in an increasing order) according to their initial starting points, i.e., $u_{i}(0) \leq$ $u_{i+1}(0)$ for all $i=1, \ldots, n-1$. Let $W^{1}, W^{2} \in \mathbb{R}_{++}$be such that $W=W^{1}+W^{2}$ and let $e^{1}=\left(N, u, W^{1}\right) \in \mathcal{E}$. For each $i \in N$, define $\widehat{u}_{i}$ by $\widehat{u}_{i}(x)=u_{i}\left(x+C O E_{i}\left(e^{1}\right)\right)$ for all $x \in \mathbb{R}_{+}$, 
and let $e^{2}=\left(\widehat{N}, \widehat{u}, W^{2}\right) \in \mathcal{E}$, where $\widehat{u}=\left(\widehat{u}_{i}\right)_{i \in N}$. Let $\sigma_{i}=u_{i}^{-1}$ and $\widehat{\sigma}_{i}=\widehat{u}_{i}^{-1}$ for each $i \in N$. Then,

$$
\operatorname{COE}_{i}(e)= \begin{cases}\sigma_{i}(\lambda) & \text { for all } i=1, \ldots, k \\ 0 & \text { for all } i=k+1, \ldots, n\end{cases}
$$

where $\lambda$ and $k$ are such that

$$
\sum_{i=1}^{k} \sigma_{i}(\lambda)=W, \text { and } u_{k+1}(0)>\lambda \geq u_{k}(0) .
$$

Similarly,

$$
\operatorname{COE}_{i}\left(e^{1}\right)= \begin{cases}\sigma_{i}\left(\lambda_{1}\right) & \text { for all } i=1, \ldots, k_{1} \\ 0 & \text { for all } i=k_{1}+1, \ldots, n\end{cases}
$$

where $\lambda_{1}$ and $k_{1}$ are such that

$$
\sum_{i=1}^{k_{1}} \sigma_{i}\left(\lambda_{1}\right)=W^{1}, \text { and } u_{k_{1}+1}(0)>\lambda_{1} \geq u_{k_{1}}(0) .
$$

Thus, note that $k \geq k_{1}$ and $\lambda \geq \lambda_{1}$. Finally,

$$
\operatorname{COE}_{i}\left(e^{2}\right)= \begin{cases}\widehat{\sigma}_{i}\left(\lambda_{2}\right) & \text { for all } i=1, \ldots, k_{2} \\ 0 & \text { for all } i=k_{2}+1, \ldots, n\end{cases}
$$

where $\lambda_{2}$ and $k_{2}$ are such that

$$
\sum_{i=1}^{k_{2}} \widehat{\sigma}_{k_{2}}\left(\lambda_{2}\right)=W^{2} \text {, and } \widehat{u}_{k_{2}+1}(0)>\lambda_{2} \geq \widehat{u}_{k_{2}}(0) .
$$

Let $y=C O E(e)-C O E\left(e^{1}\right)$ and $z=C O E\left(e^{2}\right)$. We have to show that $y=z$. To do so, note first that

$$
\widehat{u}_{i}\left(y_{i}\right)= \begin{cases}\lambda & \text { for all } i=1, \ldots, k \\ u_{i}(0) & \text { for all } i=k+1, \ldots, n\end{cases}
$$

and

$$
\widehat{u}_{i}\left(z_{i}\right)= \begin{cases}\lambda_{2} & \text { for all } i=1, \ldots, k_{2} \\ \widehat{u}_{i}(0) & \text { for all } i=k_{2}+1, \ldots, n\end{cases}
$$

Note also that, as $y$ is a feasible allocation for the economy $e^{2}$, it follows, by definition of $C O E$, that $\left(\widehat{u}_{i}\left(z_{i}\right)\right)_{i \in N}$ lexicographically dominates $\left(\widehat{u}_{i}\left(y_{i}\right)\right)_{i \in N}$. This implies that $\lambda \leq \lambda_{2}$. Thus, $k_{1} \leq k \leq k_{2}$. Then,

$$
y_{i}= \begin{cases}\sigma_{i}(\lambda)-\sigma_{i}\left(\lambda_{1}\right) & \text { for all } i=1, \ldots, k_{1} \\ \sigma_{i}(\lambda) & \text { for all } i=k_{1}+1, \ldots, k \\ 0 & \text { for all } i=k+1, \ldots, n\end{cases}
$$


and

$$
z_{i}= \begin{cases}\widehat{\sigma}_{i}\left(\lambda_{2}\right) & \text { for all } i=1, \ldots, k_{2} \\ 0 & \text { for all } i=k_{2}+1, \ldots, n\end{cases}
$$

Let $i=1, \ldots, k_{1}$. Then, $\widehat{u}_{i}(x)=u_{i}\left(x+\sigma_{i}\left(\lambda_{1}\right)\right)$ for all $x \in \mathbb{R}_{+}$. Thus, $\widehat{\sigma}_{i}(x)=\sigma_{i}(x)-\sigma_{i}\left(\lambda_{1}\right)$ for all $x \in \mathbb{R}_{+}$. In particular, $\widehat{\sigma}_{i}\left(\lambda_{2}\right)=\sigma_{i}\left(\lambda_{2}\right)-\sigma_{i}\left(\lambda_{1}\right)$. Similarly, $\widehat{\sigma}_{i}\left(\lambda_{2}\right)=\sigma_{i}\left(\lambda_{2}\right)$ for all $i=k_{1}+1, \ldots, k_{2}$. Thus, $z_{i} \geq y_{i}$ for all $i \in N$. Now, if $\lambda<\lambda_{2}$, we would have $W^{2}=\sum_{i \in N} z_{i}>$ $\sum_{i \in N} y_{i}=W^{2}$, a contradiction. Thus, it follows that $\lambda=\lambda_{2}$ and, therefore, that $k=k_{2}$, which implies that $y=z$, as desired.

We conclude by showing that no other rule within the family $\left\{E^{\varphi}\right\}_{\varphi \in \Phi}$ satisfies $C P$.

Let $\widehat{\Phi}$ denote the residual of $\Phi$ after removing the functions giving rise to $R E$ and $C O E$. Let $\varphi \in \widehat{\Phi}$. Then, there exists $\left(x_{0}, y_{0}\right) \in \mathbb{R}_{++}^{2}$ such that $\varphi\left(x_{0}^{\prime}, y_{0}\right)<\varphi\left(x_{0}, y_{0}\right)$ for all $x_{0}^{\prime}<x_{0}$. We distinguish two cases.

Case 1. $\varphi(0, y)>0$ for some $y \in \mathbb{R}_{++}$.

Let $y_{1}$ be such that there exists some $\delta>0$ for which $\varphi\left(0, y_{1}\right)=\varphi\left(x_{0}-\delta, y_{0}\right)>0$. Let $u_{1}: \mathbb{R}_{+} \rightarrow \mathbb{R}_{+}$be such that $u_{1}(x)=\frac{y_{0}}{x_{0}} x$ for all $x \in \mathbb{R}_{+}$and $u_{2}: \mathbb{R}_{+} \rightarrow \mathbb{R}_{+}$be such that $u_{2}(x)=\frac{y_{0}}{x_{0}} x+y_{1}$ for all $x \in \mathbb{R}_{+}$. Let $\varepsilon \in \mathbb{R}_{++}$be such that $\varphi\left(\varepsilon, \frac{y_{0}}{x_{0}} \varepsilon\right)<\varphi\left(0, y_{1}\right)$ and consider the economy $e^{1}=\left(\{1,2\},\left(u_{1}, u_{2}\right), \varepsilon\right)$. Then, it is straightforward to show that

$$
E^{\varphi}\left(e^{1}\right)=(\varepsilon, 0)
$$

Let $\lambda=\psi_{1}\left(x_{0}\right)=\varphi\left(x_{0}, y_{0}\right)>0$ and $W=\psi_{1}^{-1}(\lambda)+\psi_{2}^{-1}(\lambda)$. Then, it is straightforward to show that

$$
E^{\varphi}(e)=\left(\psi_{1}^{-1}(\lambda), \psi_{2}^{-1}(\lambda)\right)
$$

Finally, let $W_{2}=W-\varepsilon>0 .^{7}$ For $i=1,2$, let $\widehat{u}_{i}: \mathbb{R}_{+} \rightarrow \mathbb{R}_{+}$be such that $\widehat{u}_{i}(x)=u_{i}\left(x+E_{i}^{\varphi}\left(e^{1}\right)\right)$ and consider the economy $e^{2}=\left(\{\widehat{1}, \widehat{2}\},\left(\widehat{u}_{1}, \widehat{u}_{2}\right), W^{2}\right)$. For $i=1,2$, let $\hat{\psi}_{i}: \mathbb{R}_{+} \rightarrow \mathbb{R}_{+}$be such that $\hat{\psi}_{i}(w)=\varphi\left(w, \widehat{u}_{i}(w)\right)$ for all $w \in \mathbb{R}_{+}$. We distinguish two subcases.

Subcase 1.1. $E^{\varphi}\left(e^{2}\right)=\left(W_{2}, 0\right)$.

If so, $E^{\varphi}(e)=E^{\varphi}\left(e^{1}\right)+E^{\varphi}\left(e^{2}\right)$ if and only if $\psi_{2}^{-1}(\lambda)=0$, which would imply $\varphi\left(x_{0}, y_{0}\right)=$ $\lambda=\varphi\left(0, y_{1}\right)=\varphi\left(x_{0}-\delta, y_{0}\right)$, a contradiction.

Subcase 1.2. $E^{\varphi}\left(e^{2}\right)=\left(\hat{\psi}_{1}^{-1}\left(\lambda^{\prime}\right), \hat{\psi}_{2}^{-1}\left(\lambda^{\prime}\right)\right)$.

\footnotetext{
${ }^{7}$ Note that $\varepsilon$ can be small enough.
} 
If so, $E^{\varphi}(e)=E^{\varphi}\left(e^{1}\right)+E^{\varphi}\left(e^{2}\right)$ if and only if $\psi_{2}^{-1}(\lambda)=\hat{\psi}_{2}^{-1}\left(\lambda^{\prime}\right)$ and $\psi_{1}^{-1}(\lambda)=\varepsilon+\hat{\psi}_{1}^{-1}\left(\lambda^{\prime}\right)$. From the former equality, it follows that $\lambda=\lambda^{\prime}$, as $\hat{\psi}_{2} \equiv \psi_{2}$ is a strictly increasing function. Thus, from the latter equality, it follows that $x_{0}=\varepsilon+\hat{\psi}_{1}^{-1}(\lambda)$, or equivalently, $\varphi\left(x_{0}, y_{0}\right)=\lambda=$ $\hat{\psi}_{1}\left(x_{0}-\varepsilon\right)=\varphi\left(x_{0}-\varepsilon, \widehat{u}_{1}\left(x_{0}-\varepsilon\right)\right)=\varphi\left(x_{0}-\varepsilon, u_{1}\left(x_{0}\right)\right)=\varphi\left(x_{0}-\varepsilon, y_{0}\right)$, a contradiction.

Case 2. $\varphi(0, y)=0$ for all $y \in \mathbb{R}_{+}$.

As $\varphi \in \widehat{\Phi}$, we know that there exist $\delta_{1}, \delta_{2}>0$ such that $\varphi\left(x_{0}+\delta_{1}, y_{0}-\delta_{2}\right)=\varphi\left(x_{0}, y_{0}\right)$. It is also straightforward to show that there exists $\alpha>0$ for which $\varphi\left(x_{0}+\delta_{1}-\alpha, y_{0}-\delta_{2}\right) \neq$ $\varphi\left(x_{0}-\alpha, y_{0}\right)$.

Let $u_{1}: \mathbb{R}_{+} \rightarrow \mathbb{R}_{+}$and $u_{2}: \mathbb{R}_{+} \rightarrow \mathbb{R}_{+}$be such that

- $u_{1}\left(x_{0}\right)=y_{0}$

- $u_{2}\left(x_{0}+\delta_{1}\right)=y_{0}-\delta_{2}$

- $u_{1}(\alpha)=u_{2}(\alpha)$

Let $W \in \mathbb{R}_{+}$be such that $W>2 \alpha$. Then, consider the economies $e^{1}=\left(\{1,2\},\left(u_{1}, u_{2}\right), 2 \alpha\right)$ and $e=\left(\{1,2\},\left(u_{1}, u_{2}\right), W\right)$. It is straightforward to show that

$$
E^{\varphi}\left(e^{1}\right)=(\alpha, \alpha)
$$

and

$$
E^{\varphi}(e)=\left(\psi_{1}^{-1}(\lambda), \psi_{2}^{-1}(\lambda)\right)
$$

where $\lambda$ is such that $\psi_{1}^{-1}(\lambda)+\psi_{2}^{-1}(\lambda)=W$. Equivalently,

$$
E^{\varphi}(e)=(x, W-x)
$$

where

$$
\varphi\left(x, u_{1}(x)\right)=\varphi\left(W-x, u_{2}(W-x)\right)
$$

For $i=1,2$, let $\widehat{u}_{i}: \mathbb{R}_{+} \rightarrow \mathbb{R}_{+}$be such that $\widehat{u}_{i}(x)=u_{i}(x+\alpha)$ and consider the economy $e^{2}=\left(\{\widehat{1}, \widehat{2}\},\left(\widehat{u}_{1}, \widehat{u}_{2}\right), W^{2}\right)$, where $W^{2}=W-2 \alpha$. For $i=1,2$, let $\hat{\psi}_{i}: \mathbb{R}_{+} \rightarrow \mathbb{R}_{+}$be such that $\hat{\psi}_{i}(w)=\varphi\left(w, \widehat{u}_{i}(w)\right)$ for all $w \in \mathbb{R}_{+}$. Then,

$$
E^{\varphi}\left(e^{2}\right)=\left(\hat{\psi}_{1}^{-1}\left(\lambda^{\prime}\right), \hat{\psi}_{2}^{-1}\left(\lambda^{\prime}\right)\right)
$$

where $\lambda^{\prime}$ is such that $\psi_{1}^{-1}\left(\lambda^{\prime}\right)+\psi_{2}^{-1}\left(\lambda^{\prime}\right)=W^{2}$. Equivalently,

$$
E^{\varphi}\left(e^{2}\right)=(y, W-2 \alpha-y),
$$


where

$$
\varphi\left(y, u_{1}(y+\alpha)\right)=\varphi\left(W-2 \alpha-y, u_{2}(W-\alpha-y)\right) .
$$

Then, $E^{\varphi}(e)=E^{\varphi}\left(e^{1}\right)+E^{\varphi}\left(e^{2}\right)$ if and only if $\alpha+y=x$. Or, equivalently, if and only if $\psi_{1}^{-1}(\lambda)=\alpha+\hat{\psi}_{1}^{-1}\left(\lambda^{\prime}\right)$. If so,

$$
\varphi\left(x-\alpha, u_{1}(x)\right)=\varphi\left(W-x-\alpha, u_{2}(W-x)\right) .
$$

Now, if $W=2 x_{0}+\delta_{1}$, then (1) becomes

$$
\varphi\left(x, u_{1}(x)\right)=\varphi\left(2 x_{0}+\delta_{1}-x, u_{2}\left(2 x_{0}+\delta_{1}-x\right)\right)
$$

which implies $x=x_{0} \cdot{ }^{8}$ Thus, (2) becomes

$$
\varphi\left(x_{0}-\alpha, y_{0}\right)=\varphi\left(x_{0}+\delta_{1}-\alpha, y_{0}-\delta_{2}\right)
$$

which represents a contradiction..$^{9}$

\section{Related literature}

Our paper can be considered as part of the rapidly expanding literature on fair allocation. Traditionally, economists have been criticized for paying too little attention to distributional questions. There now exists, however, a well-developed literature devoted to the formulation and the analysis of equity concepts that traces back to Foley (1967) and his notion of envy-free allocation. In the last few years, a variety of new solutions has been proposed and applied to a wide range of models, and a number of properties of solutions have been formulated and studied for these models (see, for instance, Fleurbaey and Maniquet, forthcoming; Thomson, forthcoming; and the literature cited therein). To a large extent, this literature has been axiomatic, taking as the departure properties of allocation rules and investigating the existence of rules satisfying various combinations of these properties. This is precisely what we do in this paper for a model of resource allocation in which agents' capabilities and starting points may differ.

The use of the axiomatic method is not a discovery of the theory of fair allocation but of the theory of bargaining initiated by Nash (1950). Bargaining theory is the axiomatic

\footnotetext{
${ }^{8}$ Recall that $\varphi\left(x_{0}, y_{0}\right)=\varphi\left(x_{0}+\delta_{1}, y_{0}-\delta_{2}\right)$.

${ }^{9}$ It is worth noting that this argument requires $W=2 x_{0}+\delta_{1}>2 \alpha$. Now, we know that $x_{0}-\alpha \geq 0$ and $x_{0}+\delta_{1}-\alpha>0$, which guarantees that $2 x_{0}+\delta_{1}>x_{0}+\alpha \geq 2 \alpha$.
} 
study of utility-allocating mechanisms acting on a domain of utility possibility sets with threat points. The applications of bargaining theory to problems of distributive justice are usually not supportable, because too much justice-relevant information is lost in the specification of the domain. This has motivated the extension of the theory to economic environments, in which an aggregate starting point vector has to be allocated between several agents with given utility functions. Our model could then be considered as a specific case in which the good to be allocated is unidimensional. This seemingly innocuous aspect makes, however, our analysis independent from the (extended) bargaining theory with economic environments, in which a key assumption is that the domain consists of all environments with any positive number of dimensions (e.g., Roemer, 1988).

Axioms similar to the ones used in this paper have also been used in different, but related, models in the literature. Instances are the division problem with single-peaked preferences (e.g., Sprumont, 1991), the taxation model (e.g., Young, 1988), the rationing model (e.g., Moulin, 2000), and the scheduling model (e.g., Moulin and Stong, 2002). In those models, peaks, incomes, claims, or demands of the agents play a crucial role in resource allocation. In our case, the starting points of agents play a sort of counterpart role. The constrained outcome-egalitarian rule studied in this paper resembles, to some extent, the uniform rule, the constrained equal awards rule, and "standard of gains" method in those models. Moulin and Stong (2002), for instance, characterize the latter by consistency, composition, and demand monotonicity (a counterpart of this axiom in the current setting might be: as starting points increase, allotments do not decrease). Hence, in addition to the common axioms, Moulin and Stong (2002) use the equity axiom of "demand monotonicity", whereas here "priority" is used instead. Somewhat relatedly, Young (1988) characterizes in the taxation model the so-called equal-sacrifice solutions by strict resource-monotonicity, composition, consistency, and strict order-preservation.

\section{$5 \quad$ Further insights}

We have analyzed a simple distribution problem in which a given amount of wealth has to be distributed among individuals possessing a capability to transform wealth into some given valued (interpersonally comparable) outcome and, possibly, different (outcome) starting points. For this simple environment, we have characterized the two focal egalitarian allocation rules that exist, i.e., the one that allocates the resource equally, and the one that makes outcome 
levels as equal as possible. We have shown that these two focal rules are the only ethical and operational procedures for allocating wealth, provided we assume that ethical means prioritarian and solidaristic, and operational means obeying the axiom of composition. In doing so, we have provided, as announced in the introduction, a common ground for the two polar answers to the question "equality of what?". In particular, our characterization result also shows that the combination of the notions of priority, solidarity, and composition is equivalent to a kind of egalitarianism, where the equality in question is either resources or outcome levels.

Our analysis provides a common justification of both resource and welfare egalitarianism. Nevertheless, it is worth mentioning that ours is not a welfarist approach, the approach usually linked to welfare egalitarianism and which maintains that the justness of a state should be a function only of the welfares, or outcomes, of the agents in that state. We take instead a resourcist approach by studying allocation mechanisms defined on a space of economic environments, where the distribution of resources can be explicitly defined. In other words, we endorse the view that information concerning the distribution of goods or resources is in general necessary to evaluate the justness of a state of the world. As we have seen, this does not preclude our obtaining welfare (in our case, outcome) egalitarianism as a result of combining some (non-welfarist) axioms. ${ }^{10}$

ln moral and political philosophy, the debate between resource egalitarians and welfare egalitarians is between those who wish to hold people responsible for the choices they make and preferences they have, after some initial equality has been guaranteed, and those who wish to hold individuals responsible for nothing about themselves. A natural extension of the model in this paper would account for individual effort decisions. As a matter of fact, the ethical axioms we use in this work would only be justified for equally-deserving individuals. The literature on compensation and responsibility (e.g., Fleurbaey, 2008) provides us with an appropriate framework for such a natural extension. In its simplest case, this literature deals with the allocation of a given amount of an external one-dimensional resource (which is not produced) among a group of individuals whose outcome achievements depend on this resource, but also on their social background (a characteristic which elicits compensation) and personal effort (a characteristic which does not elicit compensation). In the parlance of our paper, this could be interpreted as saying that individual outcome functions would be bivariate functions depending on two variables reflecting the personal effort of the individual and the amount of

\footnotetext{
${ }^{10} \mathrm{An}$ early characterization of welfare egalitarianism, also by means of resourcist axioms on a domain of economic environments, is provided in Roemer (1986).
} 
the resource she is allocated. The mappings themselves would incorporate the influence of social background on individual outcome achievements. Characterizations of allocation rules for this model exist in the literature. Therefore it would be interesting to explore whether the translation of our axioms to this context would give rise to new characterizations.

Eventually, a theory of distributive justice must, we believe, postulate a domain of economies in which effort choices by individuals (relating to education and production), as well as risk preferences and level-comparable welfare, in a multi-stage model, are described. The present analysis is a far cry from that goal. Indeed, one difficulty in the work of philosophers is that they implicitly assume all these attributes of real-world societies in their theorizing. In any case, it is clear that it would be immensely difficult to deduce formally a theory of just resource allocation on such a domain, without postulating unacceptably strong axioms, and so it is not surprising that the work of political philosophers is tentative and sketchy, by their own admission.

\section{Appendix}

\subsection{The proof of Theorem 1}

We start showing that each rule in $\left\{E^{\varphi}\right\}_{\varphi \in \Phi}$ satisfies $P R$ and $S L$.

Let $\varphi \in \Phi$ and $e=(N, u, W) \in \mathcal{E}$ be given. Let $i, j \in N$ be such that $E_{i}^{\varphi}(e)<E_{j}^{\varphi}(e)$. Suppose, by contradiction, that $u_{i}\left(E_{i}^{\varphi}(e)\right)<u_{j}\left(E_{j}^{\varphi}(e)\right)$; then $\psi_{i}\left(E_{i}^{\varphi}(e)\right)=\varphi\left(E_{i}^{\varphi}(e), u_{i}\left(E_{i}^{\varphi}(e)\right)\right)<$ $\varphi\left(E_{j}^{\varphi}(e), u_{j}\left(E_{j}^{\varphi}(e)\right)\right)=\psi_{j}\left(E_{j}^{\varphi}(e)\right)$, which contradicts the definition of $E^{\varphi}$, as $E_{j}^{\varphi}(e)>0$. It then follows that $E^{\varphi}$ satisfies $P R$.

As for $S L$, let $\varphi \in \Phi$ be given and let $e=(N, u, W) \in \mathcal{E}$ and $e^{\prime}=\left(N^{\prime}, u^{\prime}, W^{\prime}\right) \in \mathcal{E}$, be such that $N \subseteq N^{\prime}$. Let $N_{1}=\left\{i \in N: R_{i}(e)>0\right\}$.

If $E_{i}^{\varphi}(e)=E_{i}^{\varphi}\left(e^{\prime}\right)$ for all $i \in N$, there is nothing to prove.

Suppose there exists $i \in N$ such that $E_{i}^{\varphi}\left(e^{\prime}\right)>E_{i}^{\varphi}(e)$. Then, $\psi_{i}\left(E_{i}^{\varphi}\left(e^{\prime}\right)\right)>\psi_{i}\left(E_{i}^{\varphi}(e)\right)$. As $E_{i}^{\varphi}\left(e^{\prime}\right)>0$, it follows that $\psi_{j}\left(E_{j}^{\varphi}(e)\right) \leq \psi_{i}\left(E_{i}^{\varphi}(e)\right)<\psi_{i}\left(E_{i}^{\varphi}\left(e^{\prime}\right)\right) \leq \psi_{j}\left(E_{j}^{\varphi}\left(e^{\prime}\right)\right)$, for all $j \in N_{1}$. Thus, $E_{j}^{\varphi}\left(e^{\prime}\right)>E_{j}^{\varphi}(e)$, for all $j \in N_{1}$ and $E_{j}^{\varphi}\left(e^{\prime}\right) \geq E_{j}^{\varphi}(e)$, for all $j \in N$, as desired.

Suppose there exists $i \in N$ such that $E_{i}^{\varphi}\left(e^{\prime}\right)<E_{i}^{\varphi}(e)$. Then, $\psi_{i}\left(E_{i}^{\varphi}\left(e^{\prime}\right)\right)<\psi_{i}\left(E_{i}^{\varphi}(e)\right)$. Note that, in this case, $i \in N_{1}$. Then, $\psi_{j}\left(E_{j}^{\varphi}(e)\right)=\psi_{i}\left(E_{i}^{\varphi}(e)\right)>\psi_{i}\left(E_{i}^{\varphi}\left(e^{\prime}\right)\right)$, for all $j \in N_{1}$. Assume that $E_{j}^{\varphi}\left(e^{\prime}\right)>0$ (otherwise, there is nothing to prove). Then, $\psi_{j}\left(E_{j}^{\varphi}(e)\right)>\psi_{i}\left(E_{i}^{\varphi}\left(e^{\prime}\right)\right) \geq$ $\psi_{j}\left(E_{j}^{\varphi}\left(e^{\prime}\right)\right)$ and, therefore, $E_{j}^{\varphi}\left(e^{\prime}\right)<E_{j}^{\varphi}(e)$, as desired. To conclude, suppose, by contradiction, 
that there exists $j \in N \backslash N_{1}$ such that $E_{j}^{\varphi}\left(e^{\prime}\right)>E_{j}^{\varphi}(e)=0$. Then, $\psi_{j}\left(E_{j}^{\varphi}(e)\right) \geq \psi_{i}\left(E_{i}^{\varphi}(e)\right)>$ $\psi_{i}\left(E_{i}^{\varphi}\left(e^{\prime}\right)\right)=\psi_{j}\left(E_{j}^{\varphi}\left(e^{\prime}\right)\right)$, which contradicts the monotonicity of $\psi_{j}$.

We now show that if a rule satisfies $P R$ and $S L$ then it is a member of the family $\left\{E^{\varphi}\right\}_{\varphi \in \Phi}$.

Let $i \in \mathbb{I}$ and $\alpha \in \mathbb{R}_{++}$be given. Define $E(R, i, \alpha)=\left\{e \in \mathcal{E}: R_{i}(e)=\alpha\right\}$ and $C(R, i, \alpha)=$ $\left\{(x, y) \in \mathbb{R}_{+}^{2}: x=R_{j}(e) ; y=u_{j}(x)\right.$ for some $e=(N, u, W) \in E(R, i, \alpha)$ and $\left.j \in N\right\}$. For ease of notation, let $\widehat{C}(R, i, \alpha)=C(R, i, \alpha) \cap \mathbb{R}_{++}^{2} \cdot{ }^{11}$ Our aim is to show that the family of curves $\left\{C(R, i, \alpha): \alpha \in \mathbb{R}_{++}\right\}$is the isoquant map of an appropriate function $\varphi \in \Phi$ and to show from here that $R=E^{\varphi}$. To do so, we start showing some features of that family of curves.

- $C(R, i, \alpha)$ is not empty.

Let $j \in \mathbb{I}$ be such that $u_{j}(M-\alpha)>u_{i}(\alpha)$ for some $M>2 \alpha$ and consider the economies $e_{1}=\left(\{i, j\},\left(u_{i}, u_{j}\right), \alpha / 2\right)$ and $e_{2}=\left(\{i, j\},\left(u_{i}, u_{j}\right), M\right)$. It is straightforward to show that $R_{i}\left(e_{1}\right)<\alpha$ and, by PR, that $R_{i}\left(e_{2}\right)>\alpha$. Let $\Omega^{<}=\left\{W \in(0,+\infty): R_{i}\left(\{i, j\},\left(u_{i}, u_{j}\right), W\right)<\alpha\right\}$ and $\Omega^{>}=\left\{W \in(0,+\infty): R_{i}\left(\{i, j\},\left(u_{i}, u_{j}\right), W\right)>\alpha\right\}$. The above shows that $\Omega^{<} \neq \emptyset \neq \Omega^{>}$. It is obvious that $\Omega^{<} \cap \Omega^{>}=\emptyset$. We show now that both are open sets. Let $W \in \Omega^{<}$, $e=\left(\{i, j\},\left(u_{i}, u_{j}\right), W\right)$, and $\bar{\alpha}=R_{i}(e)<\alpha$. Let $\varepsilon=\frac{\alpha-\bar{\alpha}}{2}$. By $R M,(W-\varepsilon, W) \subset \Omega^{<}$. Suppose, by contradiction, that there exists $W^{*} \in(W, W+\varepsilon)$ such that $W^{*} \notin \Omega^{<}$, i.e., $R_{i}\left(e^{*}\right) \geq \alpha$, for $e^{*}=\left(\{i, j\},\left(u_{i}, u_{j}\right), W^{*}\right)$. By $R M, R_{j}\left(e^{*}\right) \geq R_{j}(e)=W-\bar{\alpha}$. Then, $W^{*}=$ $R_{j}\left(e^{*}\right)+R_{i}\left(e^{*}\right) \geq W-\bar{\alpha}+\alpha=W+2 \varepsilon$, which contradicts that $W^{*} \in(W, W+\varepsilon)$. This shows that $\Omega^{<}$is an open set. Analogously, we show that $\Omega^{>}$is an open set. If, by contradiction, we assume that $R_{i}\left(\{i, j\},\left(u_{i}, u_{j}\right), W\right) \neq \alpha$, for all $W \in \mathbb{R}_{++}$, then $\mathbb{R}_{++}=\Omega^{>} \cup \Omega^{<}$. It would then follow that $(0,+\infty)$ is not connected, which is a contradiction. Thus, we have shown that, for any $(i, \alpha) \in \mathbb{I} \times \mathbb{R}_{++}$, there exists (a two-agent) economy $e \in \mathcal{E}$ such that $R_{i}(e)=\alpha$, which shows, in particular, that $C(R, i, \alpha)$ is not empty.

- $C(R, i, \alpha)$ slopes downward to the right, i.e., if $(x, y),\left(x^{\prime}, y^{\prime}\right) \in C(R, i, \alpha)$ and $x^{\prime}>x$ then $y^{\prime} \leq y \cdot{ }^{12}$

Suppose, to the contrary, that $y^{\prime}>y$. By definition, there exist $e=(N, u, W)$ and $e^{\prime}=$ $\left(N^{\prime}, u^{\prime}, W^{\prime}\right) \in E(R, i, \alpha)$ and $j \in N, k \in N^{\prime}$ such that $(x, y)=\left(R_{j}(e), u_{j}\left(R_{j}(e)\right)\right)$ and $\left(x^{\prime}, y^{\prime}\right)=$

\footnotetext{
${ }^{11}$ Note that, for any $\alpha \in \mathbb{R}_{++}, C(R, i, \alpha) \cap\{y=0\}=\emptyset$, but it might well be the case that $C(R, i, \alpha) \cap\{x=$ $0\} \neq \emptyset$, for some $\alpha \in \mathbb{R}_{++}$.

${ }^{12}$ Strictly speaking, we should say that $C(R, i, \alpha)$ slopes downward to the right with respect to its third variable. For ease of exposition, we omit "with respect to its third variable".
} 
$\left(R_{k}\left(e^{\prime}\right), u_{k}\left(R_{k}\left(e^{\prime}\right)\right)\right)$. Let $e^{j}=\left(\{i, j\},\left(u_{i}, u_{j}\right), \alpha+x\right)$ and $e^{k}=\left(\{i, k\},\left(u_{i}, u_{k}\right), \alpha+x^{\prime}\right)$. Then, by $C Y, R\left(e^{j}\right)=(\alpha, x)$ and $R\left(e^{k}\right)=\left(\alpha, x^{\prime}\right)$. Let $e^{*}=\left(\{i, j, k\},\left(u_{i}, u_{j}, u_{k}\right), \alpha+x+x^{\prime}\right)$. By $S L$, $R\left(e^{*}\right)=\left(\alpha, x, x^{\prime}\right)$, which says, in particular, that $e^{*} \in E(R, i, \alpha)$ and $R_{j}\left(e^{*}\right)<R_{k}\left(e^{*}\right)$. Thus, $P R$ implies that $u_{j}\left(R_{j}\left(e^{*}\right)\right) \geq u_{k}\left(R_{k}\left(e^{*}\right)\right)$. However, our hypothesis says that $y=u_{j}\left(R_{j}\left(e^{*}\right)\right)<$ $u_{k}\left(R_{k}\left(e^{*}\right)\right)=y^{\prime}$, a contradiction.

- $\left\{\widehat{C}(R, i, \alpha): \alpha \in \mathbb{R}_{++}\right\}$is a collection of disjoint sets.

Suppose, by contradiction, that there exists $(x, y) \in \widehat{C}\left(R, i, \alpha_{1}\right) \cap \widehat{C}\left(R, i, \alpha_{2}\right)$. Assume, without loss of generality, that $\alpha_{1}>\alpha_{2}>0$. Let $e_{1}=\left(N_{1}, u_{1}, W_{1}\right) \in E\left(R, i, \alpha_{1}\right), e_{2}=$ $\left(N_{2}, u_{2}, W_{2}\right) \in E\left(R, i, \alpha_{2}\right)$ and $j \in N_{1}, k \in N_{2}$ such that $(x, y)=\left(R_{j}\left(e_{1}\right), u_{j}\left(R_{j}\left(e_{1}\right)\right)\right)=$ $\left(R_{k}\left(e_{2}\right), u_{k}\left(R_{k}\left(e_{2}\right)\right)\right)$. Let $\widehat{e}_{1}=\left(\{i, j\},\left(u_{i}, u_{j}\right), x+\alpha_{1}\right)$ and $\widehat{e}_{2}=\left(\{i, k\},\left(u_{i}, u_{k}\right), x+\alpha_{2}\right)$. By $C Y, R_{i}\left(\widehat{e}_{1}\right)=\alpha_{1}$ and $R_{i}\left(\widehat{e}_{2}\right)=\alpha_{2}$. By $P R$ and $R M$, there exists $W>x+\alpha_{2}$ for which $\widetilde{e}_{2}=\left(\{i, k\},\left(u_{i}, u_{k}\right), W\right) \in E\left(R, i, \alpha_{1}\right)$. By $R M$, applied to $\widehat{e}_{2}$ and $\widetilde{e}_{2}$, we know that $R_{k}\left(\widetilde{e}_{2}\right)>$ $R_{k}\left(\widehat{e}_{2}\right)=x$. Therefore, $(x, y)<\left(R_{k}\left(\widetilde{e}_{2}\right), u_{k}\left(R_{k}\left(\widetilde{e}_{2}\right)\right)\right) \in \widehat{C}\left(R, i, \alpha_{1}\right)$. This contradicts the fact that $C\left(R, i, \alpha_{1}\right)$ slopes downward to the right.

- If $\alpha_{1}>\alpha_{2}>0$ then $\widehat{C}\left(R, i, \alpha_{1}\right)$ lies above $\widehat{C}\left(R, i, \alpha_{2}\right)$, i.e.,

(i) For all $(x, y) \in \widehat{C}\left(R, i, \alpha_{2}\right)$ there exists $\left(x^{\prime}, y^{\prime}\right) \in \widehat{C}\left(R, i, \alpha_{1}\right)$ such that $(x, y)<\left(x^{\prime}, y^{\prime}\right)$.

(ii) There is no $(z, t) \in \widehat{C}\left(R, i, \alpha_{2}\right)$ and $\left(z^{\prime}, t^{\prime}\right) \in \widehat{C}\left(R, i, \alpha_{1}\right)$ such that $(z, t)<\left(z^{\prime}, t^{\prime}\right)$.

Let $j \in \mathbb{I}$ be such that $u_{j}(x)=y$ and $R\left(\{i, j\},\left(u_{i}, u_{j}\right), \alpha_{2}+x\right)=\left(\alpha_{2}, x\right)$. By $P R$ and $R M$, there exists $W^{*}>\alpha_{2}+x$ for which $R_{i}\left(\{i, j\},\left(u_{i}, u_{j}\right), W^{*}\right)=\alpha_{1}$. Let $\left(x^{\prime}, y^{\prime}\right)=\left(R_{j}\left(e^{*}\right), u_{j}\left(R_{j}\left(e^{*}\right)\right)\right)$. Then, $\left(x^{\prime}, y^{\prime}\right) \in \widehat{C}\left(R, i, \alpha_{1}\right)$. It follows from $R M$, and the fact that $u_{j}$ is strictly increasing, that $\left(x^{\prime}, y^{\prime}\right)>(x, y)$, which shows statement $(i)$. Statement $(i i)$ follows from statement $(i)$ and the fact that $C\left(R, i, \alpha_{1}\right)$ slopes downward to the right.

- If $(0, y) \in C(R, i, \alpha)$ then $\left(0, y^{\prime}\right) \in C(R, i, \alpha)$ for all $y^{\prime}>y$.

Let $j \in \mathbb{I}$ be such that $u_{j}(0)=y \geq u_{i}(\alpha)$ and $R\left(\{i, j\},\left(u_{i}, u_{j}\right), \alpha\right)=(\alpha, 0)$. Let $k \in \mathbb{I}$ be such that $u_{k}(0)=y^{\prime}>y \geq u_{i}(\alpha)$ and consider the economy $e=\left(\{i, j, k\},\left(u_{i}, u_{j}, u_{k}\right), \alpha\right)$. By $S L$, either $R(e)=(\alpha, 0,0)$, which would conclude the proof, or $R(e)=(\alpha-\varepsilon, 0, \varepsilon)$, for some $\varepsilon \leq \alpha$. In the latter case, however, we would contradict $P R$, as $u_{k}(\varepsilon)>u_{j}(0)$.

In what follows, we assume that $i$ is an agent with a null starting point, i.e., $i$ is such that $u_{i}(0)=0$. 
Our richness assumption and the above properties of $C(R, i, \alpha)$ guarantee that, for each $(x, y) \in \mathbb{R}_{++}^{2}$, there exists a unique $\alpha(x, y) \in \mathbb{R}_{++}$such that $(x, y) \in \widehat{C}(R, i, \alpha(x, y)) .{ }^{13}$ For each $y \in \mathbb{R}_{+}$, let $A(y)=\left\{\alpha \in \mathbb{R}_{+}\right.$such that $\left.(0, y) \in C(R, i, \alpha)\right\}$. We now define the function $\varphi:\left\{\mathbb{R}_{+} \times \mathbb{R}_{++}\right\} \cup\{(0,0)\} \rightarrow \mathbb{R}_{+}$, as follows:

$$
\varphi(x, y)= \begin{cases}\alpha(x, y) & \text { if }(x, y) \in \mathbb{R}_{++}^{2} \\ \sup \{A(y)\} & \text { if } x=0 \text { and } A(y) \neq \emptyset \\ 0 & \text { if } x=0 \text { and } A(y)=\emptyset\end{cases}
$$

We now describe some features of $\varphi$.

- $\varphi$ is well defined.

The only pending aspect to guarantee this feature is to show that $A(y)$ is bounded for each $y \in \mathbb{R}_{+}$. Assume otherwise. Then, there exists $y \in \mathbb{R}_{+}$such that for all $M>0$ there exists $\alpha_{M} \in A(y)$ such that $\alpha_{M}>M$. Then, there exists an economy $e=\left(\{i, j\},\left(u_{i}, u_{j}\right), W\right)$, such that $R(e)=\left(\alpha_{M}, 0\right)$ (and hence, $\left.W=\alpha_{M}\right)$. By $P R$, this implies that $u_{i}\left(\alpha_{M}\right) \leq u_{j}(0)=y$, which contradicts the fact that $u_{i}$ is unbounded.

- $\varphi$ exhibits the following monotonicity properties:

(i) If $x \in \mathbb{R}_{+}$and $x^{\prime}, y \in \mathbb{R}_{++}$are such that $x<x^{\prime}$ then $\varphi(x, y) \leq \varphi\left(x^{\prime}, y\right)$.

(ii) If $x \in \mathbb{R}_{+}$and $y, y^{\prime} \in \mathbb{R}_{++}$are such that $y<y^{\prime}$ then $\varphi(x, y) \leq \varphi\left(x, y^{\prime}\right)$.

(iii) If $x \in \mathbb{R}_{+}$and $x^{\prime}, y, y^{\prime} \in \mathbb{R}_{++}$are such that $(x, y)<\left(x^{\prime}, y^{\prime}\right)$, then $\varphi(x, y)<\varphi\left(x^{\prime}, y^{\prime}\right)$.

(iv) $\varphi(0,0)=0 \leq \varphi(x, y)$ for all $(x, y) \in \mathbb{R}_{+} \times \mathbb{R}_{++}$.

Let $x, x^{\prime}, y \in \mathbb{R}_{++}$be such that $x<x^{\prime}$. If $\varphi(x, y)>\varphi\left(x^{\prime}, y\right)$ then $\widehat{C}(R, i, \varphi(x, y))$ lies above $\widehat{C}\left(R, i, \varphi\left(x^{\prime}, y\right)\right)$. In such a case, as $\left(x^{\prime}, y\right) \in \widehat{C}\left(R, i, \varphi\left(x^{\prime}, y\right)\right)$, there exists $(z, t) \in \widehat{C}(R, i, \varphi(x, y))$ such that $\left(x^{\prime}, y\right)<(z, t)$. Then, $(z, t)>(x, y)$, which contradicts the fact that $\widehat{C}(R, i, \varphi(x, y))$ slopes downward to the right. Suppose now that $x=0$ and assume, by contradiction, that $\varphi(0, y)>\varphi(x, y)$. Let $\widehat{\alpha}=\varphi(0, y)$. As $C(R, i, \widehat{\alpha})$ slopes downward to the right, it follows that $(x, y) \in \widehat{C}(R, i, \alpha)$, for some $\alpha \geq \widehat{\alpha}$, which would imply that $\varphi(x, y) \geq \widehat{\alpha}$, a contradiction. This shows statement $(i)$.

\footnotetext{
${ }^{13}$ Let $j \in \mathbb{I}$ be such that $u_{j}(x)=y$. By $R M$ and $P R$, there exists some $W>0$ for which $R_{j}\left(\{i, j\},\left(u_{i}, u_{j}\right), W\right)=x$. By $P R, i$ can never be awarded with zero resources. Thus, $\alpha=W-x>0$, and $(x, y) \in \widehat{C}(R, i, \alpha)$.
} 
As for statement $(i i)$, let $x, y, y^{\prime} \in \mathbb{R}_{++}$be such that $y<y^{\prime}$. If $\varphi(x, y)>\varphi\left(x, y^{\prime}\right)$ then $\widehat{C}(R, i, \varphi(x, y))$ lies above $\widehat{C}\left(R, i, \varphi\left(x, y^{\prime}\right)\right)$. In such a case, as $\left(x, y^{\prime}\right) \in \widehat{C}\left(R, i, \varphi\left(x, y^{\prime}\right)\right)$, there exists $(z, t) \in \widehat{C}(R, i, \varphi(x, y))$ such that $\left(x, y^{\prime}\right)<(z, t)$. Then, $(z, t)>(x, y)$, which contradicts the fact that $\widehat{C}(R, i, \varphi(x, y))$ slopes downward to the right. Suppose now that $x=0$. In such a case, and as $A(y) \subset A\left(y^{\prime}\right)$, it follows that $\varphi(0, y)=\sup \{A(y)\} \leq \sup \left\{A\left(y^{\prime}\right)\right\}=\varphi\left(0, y^{\prime}\right)$. This shows statement $(i i)$.

As for statement $(i i i)$, let $x, x^{\prime}, y, y^{\prime} \in \mathbb{R}_{++}$be such that $(x, y)<\left(x^{\prime}, y^{\prime}\right)$. As each $C(R, i, \alpha)$ slopes downward to the right, it follows that $\varphi(x, y) \neq \varphi\left(x^{\prime}, y^{\prime}\right)$. If, by contradiction, $\varphi(x, y)>$ $\varphi\left(x^{\prime}, y^{\prime}\right)$ then $\widehat{C}(R, i, \varphi(x, y))$ lies above $\widehat{C}\left(R, i, \varphi\left(x^{\prime}, y^{\prime}\right)\right)$. We have, however, that $(x, y) \in$ $\widehat{C}(R, i, \varphi(x, y)),\left(x^{\prime}, y^{\prime}\right) \in \widehat{C}\left(R, i, \varphi\left(x^{\prime}, y^{\prime}\right)\right)$ and $(x, y)<\left(x^{\prime}, y^{\prime}\right)$, which represents a contradiction. Assume now that $x=0$. As before, the fact that each $C(R, i, \alpha)$ slopes downward to the right guarantees that $\varphi(0, y) \neq \varphi\left(x^{\prime}, y^{\prime}\right)$. Assume, by contradiction, that $\varphi(0, y)>\varphi\left(x^{\prime}, y^{\prime}\right)$. Let $\alpha_{1}=\varphi(0, y)$ and $\alpha_{2}=\varphi\left(x^{\prime}, y^{\prime}\right)$. Then, $\left(x^{\prime}, y^{\prime}\right) \in \widehat{C}\left(R, i, \alpha_{2}\right)$. As $C(R, i, \alpha)$ slopes downward to the right, there exist $\varepsilon>0$ sufficiently small, and $y_{\varepsilon} \leq y$ such that $\left(\varepsilon, y_{\varepsilon}\right) \in \widehat{C}\left(R, i, \alpha_{1}\right)$, which contradicts the fact that $\widehat{C}\left(R, i, \alpha_{1}\right)$ lies above $\widehat{C}\left(R, i, \alpha_{2}\right)$. This shows statement (iii). Finally, statement $(i v)$ follows from the fact that $A(0)=\emptyset$.

- $\varphi$ exhibits the following continuity properties.

(i) $\lim _{(x, y) \rightarrow\left(x_{0}, y_{0}\right)} \varphi(x, y)=\varphi\left(x_{0}, y_{0}\right)$, for all $\left(x_{0}, y_{0}\right) \in \mathbb{R}_{++}^{2}$.

(ii) $\lim _{x \rightarrow 0^{+}} \varphi(x, y(x))=\varphi\left(0, y_{0}\right)$, for all $y_{0} \in \mathbb{R}_{+}$and $y: \mathbb{R}_{++} \rightarrow \mathbb{R}_{++}$such that $\lim _{x \rightarrow 0^{+}} y(x)=$ $y_{0}$.

Let $\left(x_{0}, y_{0}\right) \in \mathbb{R}_{++}^{2}$ and let $\left\{\left(x_{n}, y_{n}\right)\right\} \rightarrow\left(x_{0}, y_{0}\right)$. In order to prove statement $(i)$, it suffices to show that $\left\{\alpha_{n}\right\}=\left\{\varphi\left(x_{n}, y_{n}\right)\right\} \rightarrow \alpha=\varphi\left(x_{0}, y_{0}\right)$. If such is not the case, then there exists a subsequence $\left\{\alpha_{k_{n}}\right\}$ that converges to $\bar{\alpha} \neq \alpha$. We assume, without loss of generality, that $\bar{\alpha}<\alpha$. Then, for $k_{n}$ sufficiently large, $\alpha_{k_{n}}<\frac{\alpha+\bar{\alpha}}{2}<\alpha$, and therefore, $\widehat{C}\left(F, i, \alpha_{k_{n}}\right)$ lies below $\widehat{C}\left(F, i, \frac{\alpha+\bar{\alpha}}{2}\right)$ and this one lies below $\widehat{C}(F, i, \alpha)$. In particular, there exists a ball $B$, about $(x, y) \in \widehat{C}(F, i, \alpha)$, which lies above $\widehat{C}\left(F, i, \frac{\alpha+\bar{\alpha}}{2}\right)$. As $\left(x_{n}, y_{n}\right) \rightarrow\left(x_{0}, y_{0}\right)$, it follows that, for large $k_{n},\left(x_{k_{n}}, y_{k_{n}}\right) \in B$. On the other hand, $\left(x_{k_{n}}, y_{k_{n}}\right) \in \widehat{C}\left(F, i, \alpha_{k_{n}}\right)$ which, for large $k_{n}$, lies below $\widehat{C}\left(F, i, \frac{\alpha+\bar{\alpha}}{2}\right)$. This represents a contradiction.

As for statement $(i i)$, it suffices to replicate the previous argument replacing $y_{0}$ by $0, y_{n}$ by $y\left(x_{n}\right) \in \mathbb{R}_{++}$, and considering $B \cap \mathbb{R}_{++}^{2}$ instead of $B$. 
The proof of this statement of the theorem concludes by showing that $R=E^{\varphi}$, i.e., $R(N, u, W)=E^{\varphi}(N, u, W)$ for all $(N, u, W) \in \mathcal{E}$.

Fix $e=(N, u, W) \in \mathcal{E}$. Two cases are distinguished.

Case 1: $i \in N$. Let $\lambda=R_{i}(e)>0$. Then, $\left(R_{j}(e), u_{j}\left(R_{j}(e)\right)\right) \in C(R, i, \lambda)$ for all $j \in N$. Thus, $\psi_{j}\left(R_{j}(e)\right)=\lambda$ for all $j \in N$ such that $R_{j}(e)>0$ and $\psi_{j}\left(R_{j}(e)\right) \geq \lambda$ for all $j \in N$ such that $R_{j}(e)=0$. As $\sum_{j \in N} R_{j}(e)=W$, it follows that $R(e)=E^{\varphi}(e)$.

Case 2: $i \notin N$. Let $j \in N$ be such that $R_{j}(e)>0$ and denote $w_{j}=R_{j}(e)$. By $P R$ and $R M$, there exists $w_{i}>0$ such that $R(\widehat{e})=\left(w_{i}, w_{j}\right)$, where $\widehat{e}=\left(\{i, j\},\left(u_{i}, u_{j}\right), w_{i}+w_{j}\right)$. We show that $C\left(R, j, w_{j}\right) \subseteq C\left(R, i, w_{i}\right)$. To do so, let $(x, y) \in C\left(R, j, w_{j}\right)$. Then, there exists $l \in \mathbb{I}$ such that $y=u_{l}(x)$ and $\left(w_{j}, x\right)=\left(R_{j}\left(e^{2}\right), R_{l}\left(e^{2}\right)\right)$, where $e^{2}=\left(\{j, l\},\left(u_{j}, u_{l}\right), w_{j}+x\right)$. Then, by $S L, R\left(e^{3}\right)=\left(w_{i}, w_{j}, x\right)$, where $e^{3}=\left(\{i, j, l\},\left(u_{i}, u_{j}, u_{l}\right), w_{i}+w_{j}+x\right)$. Consequently, $(x, y) \in C\left(R, i, w_{i}\right)$, as desired. We now distinguish two subcases.

Subcase 2.1: $R_{l}(e)=0$ for all $l \in N \backslash\{j\}$. In other words, $W=w_{j}$. Then, as $\left(0, u_{l}(0)\right)=$ $\left(R_{l}(e), u_{l}\left(R_{l}(e)\right)\right) \in C\left(R, j, w_{j}\right)$ for all $l \in N \backslash\{j\}$, the above shows that $\left(R_{l}(e), u_{l}\left(R_{l}(e)\right)\right) \in$ $C\left(R, i, w_{i}\right)$ for all $l \in N$. Thus, $\psi_{j}(W)=w_{i} \leq \psi_{l}(0)$ for all $l \in N \backslash\{j\}$, which implies that $E^{\varphi}(e)=\left(0, \ldots, 0, \underset{j^{\text {th }} \text { allocation }}{W}, 0, \ldots, 0\right)=R(e)$.

Subcase 2.2: $R_{k}(e)>0$ for some $k \in N \backslash\{j\}$. Denote $w_{k}=R_{k}(e)>0$. As above, by $P R$ and $R M$, there exists $\widetilde{w}_{i}>0$ such that $R(\widetilde{e})=\left(\widetilde{w}_{i}, w_{k}\right)$, where $\widetilde{e}=\left(\{i, k\},\left(u_{i}, u_{k}\right), \widetilde{w}_{i}+w_{k}\right)$. Analogously to the above argument, we show that $C\left(R, k, w_{k}\right) \subseteq C\left(R, i, \widetilde{w}_{i}\right)$. Thus, it follows that $\left(w_{j}, u_{j}\left(w_{j}\right)\right) \in \widehat{C}\left(R, i, w_{i}\right) \cap \widehat{C}\left(R, i, \widetilde{w}_{i}\right)$, which implies that $w_{i}=\widetilde{w}_{i}$. Let $\lambda=w_{i}=\widetilde{w}_{i}$. Then, $\left(R_{l}(e), u_{l}\left(R_{l}(e)\right)\right) \in C(R, i, \lambda)$ for all $l \in N$. From here, the proof of Case 1 concludes.

\subsection{Auxiliary statement}

A rule satisfies solidarity if and only if it satisfies consistency and resource monotonicity.

Proof. Let $R$ be a rule satisfying $S L$. We show that $R$ satisfies $R M$ and $C Y$.

Let $e=(N, u, W)$ and $e^{\prime}=\left(N, u, W^{\prime}\right) \in \mathcal{E}$ be such that $W^{\prime}<W$. Let $N_{1}=\{i \in N$ : $\left.R_{i}(e)>0\right\}$. As $\sum_{i \in N} R(e)=W>W^{\prime}=\sum_{i \in N} R\left(e^{\prime}\right)$, it follows, by $S L$, that $R_{i}\left(e^{\prime}\right) \leq R_{i}(e)$, for all $i \in N$, and $R_{i}\left(e^{\prime}\right)<R_{i}(e)$, for all $i \in N_{1}$, which shows that $R$ satisfies $R M$.

As for $C Y$, let $e=(N, u, W) \in \mathcal{E}$ and $N^{\prime} \subset N$ such that $e^{\prime}=\left(N^{\prime}, u^{\prime}, W^{\prime}\right) \in \mathcal{E}$, where $W^{\prime}=\sum_{i \in N^{\prime}} R_{i}(e)$. Suppose, by contradiction, that there exists $j \in N^{\prime}$ such that $R_{j}(e) \neq$ 
$R_{j}\left(e^{\prime}\right)$. Then, by SL, either $W^{\prime}=\sum_{i \in N^{\prime}} R_{i}(e)<\sum_{i \in N^{\prime}} R_{i}\left(e^{\prime}\right)=W^{\prime}$, or $W^{\prime}=\sum_{i \in N^{\prime}} R_{i}(e)>$ $\sum_{i \in N^{\prime}} R_{i}\left(e^{\prime}\right)=W^{\prime}$, a contradiction in any case.

Conversely, let $R$ be a rule satisfying $R M$ and $C Y$. Let $e=(N, u, W) \in \mathcal{E}$ and $e^{\prime}=$ $\left(N^{\prime}, u^{\prime}, W^{\prime}\right) \in \mathcal{E}$, be such that $N \subseteq N^{\prime}$. Let $N_{1}=\left\{i \in N: R_{i}(e)>0\right\}$. Consider the auxiliary economy $\widehat{e}=(N, u, \widehat{W}) \in \mathcal{E}$, where $\widehat{W}=\sum_{i \in N} R_{i}\left(e^{\prime}\right)$. Then, by $C Y, R(\widehat{e})=R_{N}\left(e^{\prime}\right) .{ }^{14}$ By $R M$, one of the following three possibilities happens:

$$
\begin{gathered}
\qquad R(\widehat{e})=R(e), \\
R_{i}(\widehat{e}) \leq R_{i}(e), \text { for all } i \in N \text {, and } R_{i}\left(e^{\prime}\right)<R_{i}(e) \text {, for all } i \in N_{1}, \\
R_{i}(\widehat{e}) \geq R_{i}(e), \text { for all } i \in N, \text { and } R_{i}\left(e^{\prime}\right)>R_{i}(e) \text {, for all } i \in N_{1} .
\end{gathered}
$$

Altogether, we have that $R$ satisfies $S L$.

\section{References}

[1] Chun, Y. (1999), Equivalence of axioms for bankruptcy problems, International Journal of Game Theory 28, 511-520.

[2] Dworkin, R. (1981a), What is equality? Part 1: Equality of welfare. Philosophy \& Public Affairs 10, 185-246.

[3] Dworkin, R. (1981b), What is equality? Part 2: Equality of resources. Philosophy \& Public Affairs 10, 283-345.

[4] Fleurbaey, M., (2008), Fairness, Responsibility, and Welfare, Oxford University Press.

[5] Fleurbaey, M., Maniquet, F., (forthcoming), A Theory of Fairness and Social Welfare, Econometric Society Monograph, Cambridge University Press.

[6] Foley, D., (1967), Resource allocation and the public sector, Yale Economic Studies 7, 43-98.

[7] Kalai, E., (1977), Proportional solutions to bargaining situations: Interpersonal utility comparisons, Econometrica 45, 1623-30.

\footnotetext{
${ }^{14}$ Note that the bilateral version of the consistency axiom, in which $\left|N^{\prime}\right|=2$, would not be enough.
} 
[8] Maniquet, F., Sprumont, Y., (2010), Sharing the cost of a public good: An incentiveconstrained axiomatic approach, Games and Economic Behavior 68, 275-302

[9] Moreno-Ternero J., Roemer J., (2006), Impartiality, priority, and solidarity in the theory of justice, Econometrica 74, 1419-1427

[10] Moulin H., (2000), Priority rules and other asymmetric rationing methods. Econometrica 68, 643-684.

[11] Moulin, H., Stong R., (2002), Fair Queuing and other Probabilistic Allocation Methods, Mathematics of Operations Research 27, 1-30.

[12] Nash, J., (1950) The bargaining problem, Econometrica 28, 155-162.

[13] Rawls, J. (1971), A Theory of Justice. Harvard University Press. Cambridge, MA.

[14] Roemer, J., (1986), Equality of resources implies equality of welfare, Quarterly Journal of Economics 101, 751-784.

[15] Roemer, J., (1988), Axiomatic bargaining theory in economic environments, Journal of Economic Theory 45, 1-31.

[16] Sen, A., (1973), On Economic Inequality, Clarendon Press, Oxford.

[17] Sen, A., (1980), Equality of what? In Tanner Lectures on Human Values, Vol. I. Cambridge University Press.

[18] Sprumont, Y., (1991), The division problem with single-peaked preferences: a characterization of the uniform rule, Econometrica 59, 509-519.

[19] Thomson, W., (1983), Equity in exchange economies. Journal of Economic Theory 29, 217-44.

[20] Thomson, W., (2007), Consistent Allocation Rules, Book Manuscript. University of Rochester.

[21] Thomson, W., (forthcoming), The Theory of Fair Allocation. Princeton University Press.

[22] Young P., (1988), Distributive justice in taxation, Journal of Economic Theory 44, 321-335. 